\title{
PhoA gene fusions in Legionella pneumophila generated in vivo using a new transposon, MudphoA
}

\author{
Mark A. Albano, ${ }^{1}$ Juan Arroyo, ${ }^{1}$ Barry I. Eisenstein ${ }^{1,2}$ \\ and N. Cary Engleberg ${ }^{1,2 *}$ \\ ${ }^{1}$ Department of Microbiology and Immunology, and \\ ${ }^{2}$ Department of Internal Medicine, University of Michigan \\ Medical School, Ann Arbor, Michigan 48109, USA.
}

\section{Summary}

To enable effective use of phoA gene fusions in Legionella pneumophila, we constructed MudphoA, a derivative of the mini-Mu phage Mu dll4041, which is capable of generating gene fusions to the Escherichia coli alkaline phosphatase gene (EC 3.1.3.1). Although an existing fusion-generating transposon, TnphoA, has been a useful tool for studying secreted proteins in other bacteria, this transposon and other Tn5 derivatives transpose inefficiently in Legionella pneumophila, necessitating the construction of a more effective vector for use in this pathogen. Using MudphoA we generated fusions to an $E$. coli gene encoding a periplasmic protein and to an L. pneumophila gene encoding an outer membrane protein; both sets of fusions resulted in alkaline phosphatase activity. We have begun to use MudphoA to mutate secreted proteins of L. pneumophila specifically, since this subset of bacterial proteins is most likely to be involved in host-bacterial interactions. This modified transposon may be useful for studies of other bacteria that support transposition of $\mathrm{Mu}$, but not Tn5, derivatives.

\section{Introduction}

Bacterial proteins that mediate the virulence of pathogens are most likely to be expressed at the bacterial surface or exported from the bacterial cell. In the case of Legionella pneumophila, several secreted (or exported) proteins with potential roles in pathogenesis have been identified. These include Mip, a $24 \mathrm{kDa}$ surface protein (Cianciotto et al., 1989), MSP, a $38 \mathrm{kDa}$ protease (Dreyfus and Iglewski, 1986), and legiolysin, a $39 \mathrm{kDa}$ protein that confers haemolysis, pigment production and fluorescence activities (Williams et al., 1991). Several genetic methods

Received 13 August, 1991; revised 17 February, 1992; accepted 18 March, 1992. *For correspondence. Tel. (313) 936 0879; Fax (313) 764 3562. for mutational analysis in L. pneumophila have been reported. Among these methods are allelic exchange of mutant for wild-type genes mediated by tri-parental conjugational matings, introduction of Tn5 on the broad-hostrange plasmid pRK340, and introduction of bacteriophage $\mathrm{Mu}$ on the broad-host-range plasmid pRK2 (Cianciotto et al., 1988; Dreyfus and Iglewski, 1985; Keen et al., 1985; Mintz and Shuman, 1987).

One way of identifying genes encoding proteins that are localized specifically to the bacterial envelope or periplasm is by using transposons that generate fusions between host proteins and enzymes that are active only when secreted. For example, TnphoA generates fusions to alkaline phosphatase and TnblaM to $\beta$-lactamase, both normally periplasmic proteins in Escherichia coli (Manoil and Beckwith, 1985; Broome-Smith et al., 1990). We found that TnphoA functions poorly as an insertional mutagen in L. pneumophila. Alternatively, Mu transposons are known to be functional in this bacterium (Mintz and Shuman, 1987). We therefore decided to modify Mu dll 4041 by ligating a phoA gene fragment near its right end, thereby constructing Mu dllphoA (herein called MudphoA). Mu dll4041 was chosen because it contains a $\mathrm{BamHI}$ site 116 base pairs (bp) from the Mu right end and ligations into this site are capable of maintaining an open reading frame from the $\mathrm{Mu}$ right end through the ligated fragment (Castilho et al., 1984; Kahmann and Kamp, 1979). Similar mini-Mu (Ap,lac) transposons have been constructed for in vivo generation of transcriptional and translational gene fusions by ligating an $E$. coli lac fragment into the BamHI site (Casadaban and Chou, 1984).

\section{Results}

\section{Construction of MudphoA}

We inserted the $E$. coli alkaline phosphatase gene (phoA) near the right end of mini-Mu transposon $\mathrm{Mu}$ dll4041(Castilho et al., 1984) (Fig 1). To facilitate this construction, Mudll4041 was first transposed into plasmid pUC18 18 , using strain BAC101 (Table 1). A DNA fragment encoding the phoA gene, missing amino-terminal sequences, was ligated into the $\mathrm{Bam} \mathrm{HI}$ and $\mathrm{Xhol}$ sites on pUC18 $1:$ Mudll4041. The Tn5 kanamycin resistance gene (neo) (Beck et al., 1982) was inserted adjacent to the phoA fragment. The resulting transposon, Mu dilphoA 


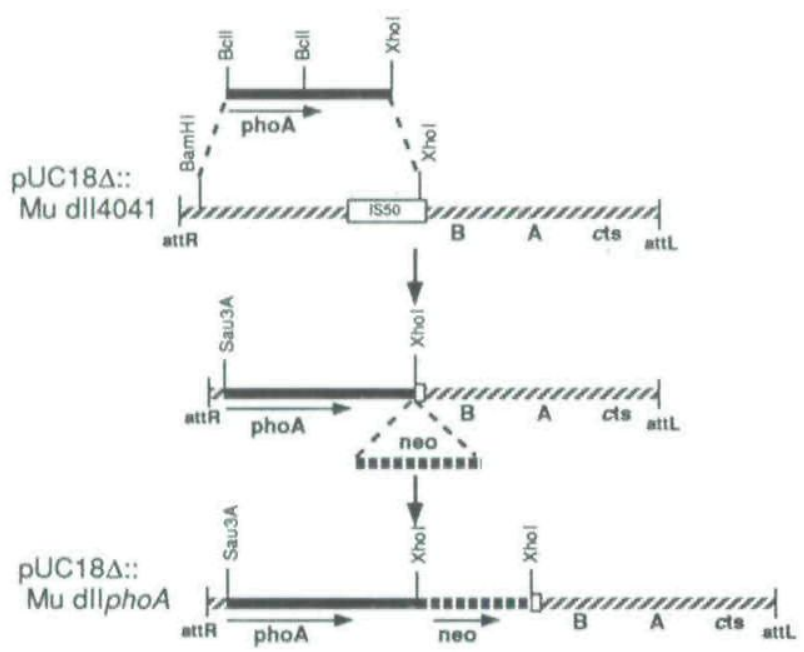

Fig. 1. Construction of MudphoA. Mu dll4041 was transposed onto pUC18 18 , a derivative of pUC18 that lacks the small Pvull fragment containing the multiple cloning sequence (Yanisch-Perron et al., 1985). The $B c / 1-X h o l$ fragment containing the E. coli alkaline phosphatase ( $p h o A$ ) gene was isolated from pSMJ11.4::TnphoA (see Table 1) and ligated to pUC184: Mu dll4041, replacing the BamHH-Xhol fragment in Mu dll4041, which contained the neo gene $\left(\mathrm{Km}^{\text {P) }}\right.$ and most of the IS50 sequences (Manoil and Beckwith, 1985). An Xhol fragment from pSMJ11.4::TnphoA containing the $\mathrm{Km}^{R}$ gene (neo) was ligated to the unique $X$ ho I site on pUC18: :MudphoA. All further work was done using the clone with the $\mathrm{Km}^{\mathrm{A}}$ gene transcribed in the same direction as the phoA gene. Mu right end and $\mathrm{Mu}$ left end refer to the right and left $\mathrm{Mu}$ terminal repeat ('attachment') sequences, respectively. $A$ and $B$ are the essential Mu transposition-replication genes and cts is the temperaturesensitive Mu repressor gene, cts62 (Casadaban and Chou, 1984).

(MudphoA), possesses only those Mu sequences necessary for transposition, it is defective for making phage particles, and it has a temperature-sensitive repressor gene (cts62).

\section{Characterization of the junction between the inside $\mathrm{Mu}$ right end and pho $\mathrm{A}$}

For MudphoA to be effective in generating protein fusions, its phoA fragment must be capable of expressing alkaline phosphatase activity, and the junction between the right end of $\mathrm{Mu}$ and $p h o A$ must maintain a translational reading frame through the $\mathrm{Mu}$ right end. The $B c$ ll-Xhol fragment from TnphoA lacks the phoA promoter, ribosome-binding site, and signal peptide sequences, as well as the coding sequence for 28 amino acids beyond the signal sequence cleavage point, to Asp-29 (Chang et al., 1986). Since Tn5-phoA junctions up to the 40 th amino acid beyond the signal cleavage point are still capable of generating normal levels of phosphatase activity (Manoil and Beckwith, 1985), we expected that the phoA fragment in MudphoA would also express the activity.
Ligation of the 5 '-end of the phoA fragment into the right end of Mu dll 4041 maintains not only the reading frame of the phoA gene but also the Sau3A restriction site at the Mu right end-phoA junction (Fig. 1). To confirm that this site was preserved, we analysed a deletion derivative of pUC18 $::$ MudphoA, since it was easier to map the Sau3A site on this smaller plasmid (Fig. 2). The presence of this junctional Sau3A site was taken as evidence that the ligation was precise and that the junction maintained the correct translational reading frame.

\section{Transposition of MudphoA and construction of a double Mu lysogen, EA17}

To test whether MudphoA would transpose as expected, strain M8820Mucts was transformed with pUC184::MudphoA and one of the transformants was purified. Plasmid

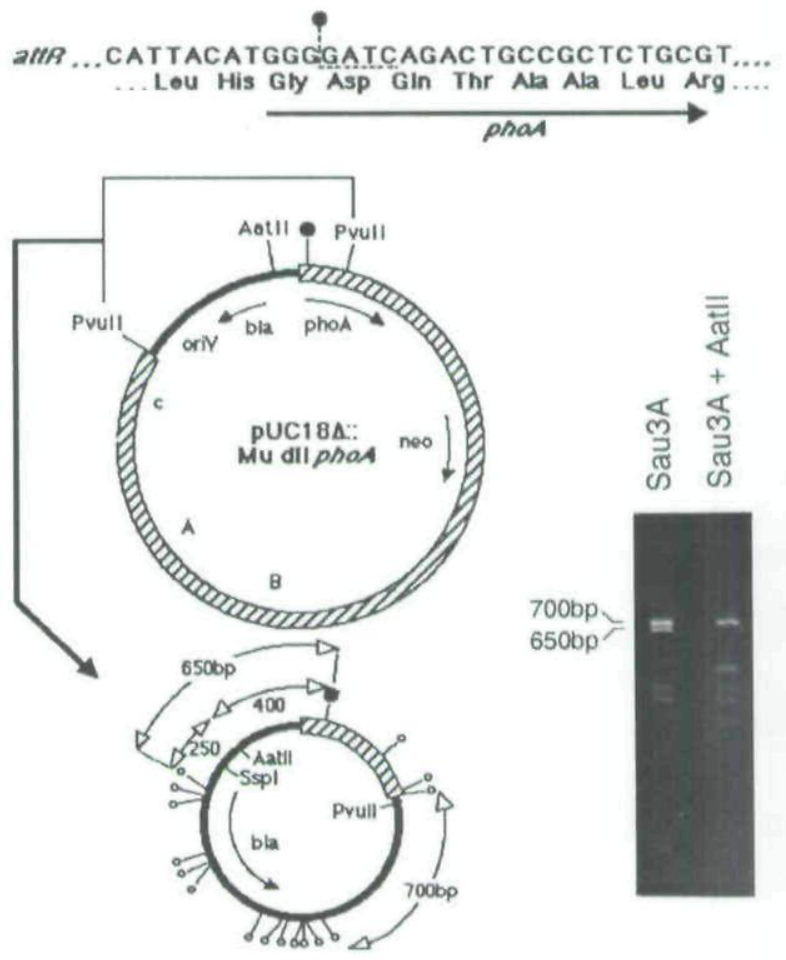

Fig. 2. Analysis of the Mu dll4041-phoA ligation junction. The inferred sequence of the junction is shown at the top of the figure with the junctional SaL3A site marked by a solid circle. pUC184::MudphoA was digested with Pvull and religation of the smaller fragment resulted in recovery of a deletion plasmid that contained the junctional Sau3A site (-) as well as several other SaLBA sites (O). The 700 bp and 650 bp fragments indicated on the circular map are shown in the restriction enzyme analysis of the pUC184:MudphoA deletion plasmid (lower right). As indicated on the map, digestion of the 650 bp Sau3A fragment with Aatll results in two smaller fragments of $400 \mathrm{bp}$ and $250 \mathrm{bp}$. Note the disappearance of the $650 \mathrm{bp}$ fragment on the gel in the double digest. Double digestion with $S$ spl and Sau3A also eliminated the 650 bp fragment and produced fragments of the expected size (data not shown). 
Table 1. Bacterial strains and plasmids.

\begin{tabular}{|c|c|c|}
\hline Strain/Plasmid & Description or Genotype & Source or Reference \\
\hline \multicolumn{3}{|l|}{ E. colli } \\
\hline M8820 & $\begin{array}{l}\mathrm{F}, \text { araD139, } \Delta(\text { ara-leu }) 7697, \\
\quad \triangle(\text { proAB-argF-laclPOZYA)XIII, rpsL }\end{array}$ & Casadaban (1975) \\
\hline $\mathrm{M} 8820 \mathrm{Mu}$ & $\begin{array}{l}\mathrm{F}, \operatorname{araD139,} \Delta(\text { ara-leu }) 7697, \Delta(\text { proAB- } \\
\text { argF-lacIPOZYA)XIII, rpsL, Mu } c^{+}\end{array}$ & Casadaban and Chou (1984) \\
\hline M8820Mucts & M8820 with Mu cts62 & Casadaban and Chou (1984) \\
\hline MG1655 & Prototroph, $\mathrm{F}, \lambda^{-}$ & Guyer et al. (1981) \\
\hline JC10240 & $\begin{array}{l}\text { Hfr, thr } 300, \text { recA56, sri300::Tn 10 }\left(\mathrm{Tc}^{\mathrm{A}}\right)^{-} \text {, } \\
\text { relA1, rlv318, spoT, thi1, rpsE2300, } \lambda^{-}\end{array}$ & Csonka and Clark (1979) \\
\hline BAC101 & $\begin{array}{l}\text { M8820 recA56 srl::Tn } 10\left(\text { Tc }^{A}\right) \text { Mu cts } 62 \text { with } \\
\text { Mu dill4041 }\end{array}$ & Casadaban and Chou (1984) \\
\hline EA4 & M8820Mucts, recA56, srl300::Tn10 $\left(\mathrm{Tc}^{\mathrm{A}}\right)$ & This work \\
\hline EA17 & EA4 with MudphoA prophage & This work \\
\hline$x 2981$ & $\begin{array}{l}\text { E. coli K-12, F' } \Delta 41\left[\text { proB-lacYZ], } \lambda^{-}\right. \\
\text {T3r, } \triangle a s d A 4, \text { zht-2::Tn10, cycA1 }\end{array}$ & R. Curtiss \\
\hline $\begin{array}{l}\chi 2981 \\
\text { (pTLP7::MudphoA) }\end{array}$ & $\chi 2981$ with $p$ TLP7::MudphoA & This work \\
\hline$\chi 2981(p R K 212.1)$ & $\chi 2981$ with pRK212.1 & J.E. Rogers \\
\hline CC118 & $\begin{array}{l}\text { araD139, } \Delta(\text { ara, leu }) 7696, \Delta \text { lacX74, } \\
\text { phoA } 120, \text { gal } E, \text { galK, thi, rps } E, r p o B, \\
\arg E_{\text {am }} \text { recA1 }\end{array}$ & Manoil and Beckwith (1985) \\
\hline \multicolumn{3}{|l|}{ L. pneumophila } \\
\hline AA100 & L. pneumophila SG1 130b & Engleberg et al. (1984a) \\
\hline AA103 & $\begin{array}{l}\text { L. pneumophila SG1 } 130 \mathrm{~b} \text { high-frequency } \\
\text { conjugation recipient }\end{array}$ & Cianciotto et al. (1988) \\
\hline AA110 & AA103 with MudphoA fusion & This work \\
\hline AA111 & AA103 with MudphoA fusion & This work \\
\hline AA112 & AA103 with MudphoA fusion & This work \\
\hline AA113 & AA103 with MudphoA fusion & This work \\
\hline AA114 & AA100 with pNC31.5: MudphoA & This work \\
\hline AA118 & AA103 with MudphoA fusion & This work \\
\hline \multicolumn{3}{|l|}{ Plasmids } \\
\hline pUC18 & $\begin{array}{l}\text { pUC18, } P v u l l \text { deletion removing the polylinker } \\
\text { region, } \mathrm{Ap}^{R}\end{array}$ & This work \\
\hline pUC184::Mu dil4041 & pUC18 18 with Mu dll 4041 insertion, $\mathrm{Ap}^{R}, \mathrm{Km}^{\mathrm{R}}$ & This work \\
\hline pUC18 $18:$ :Mud $\rho h o A$ & pUC18 $\Delta$ with MudphoA, $A p^{A} \mathrm{Km}^{\mathrm{P}}$ & This work \\
\hline pSMJ11.4::TnphoA & $A p^{R}, p a t: T n p h o A$ & Engleberg et al. (1991) \\
\hline pNC31.5 & $\mathrm{Ap}^{A}, \mathrm{Cm}^{R}$, oriT, mip & Cianciotto et al. (1989) \\
\hline PTLP7 & $\mathrm{Cm}^{R}$, sacB, oriT & This work \\
\hline pTLP7::MudphoA & $\mathrm{Cm}^{\mathrm{R}}, \mathrm{Km}^{\mathrm{R}}, \mathrm{sacB}$, oriT & This work \\
\hline pRK212.1 & $\mathrm{Ap}^{\mathrm{P}}, \mathrm{Tc}^{\mathrm{A}}, \mathrm{Tra}^{+}$ & Figurski et al. (1976) \\
\hline
\end{tabular}

DNA isolated from this strain was compared to pUC184::MudphoA by restriction enzyme digestions to ensure that pUC18 $:$ :MudphoA had been stably introduced and had not undergone any rearrangements or deletions. The restriction mapping indicated that the two plasmids were identical. M8820Mucts harbours a complete Mu prophage that is capable of making phage particles. Since it also possesses the temperature-sensitive repressor gene (cts62), phage growth and transposition are inducible at the permissive temperature of $42^{\circ} \mathrm{C}$ (Castilho et al., 1984).

A culture of M8820Mucts (pUC184::MudphoA) was grown at $30^{\circ} \mathrm{C}$ and then shifted to $42^{\circ} \mathrm{C}$ to initiate transposition and lysis. A lysate prepared from this culture was used to transduce $E$. coli strain MG1655 to kanamycin resistance $\left(\mathrm{Km}^{\mathrm{R}}\right)$. The M8820Mucts (pUC184::MudphoA) strain produced lysate titres of $10^{8}-10^{9}$ plaque-forming units (pfu) per $\mathrm{ml}$ when titred on MG1655, and the frequency of $\mathrm{Km}^{\mathrm{R}}$ transduction was $10^{-3}-10^{-4}$ per recipient cell at a multiplicity of infection of 1 . Among the $\mathrm{Km}^{\mathrm{R}}$ transductants, $97 \%$ were ampicillin sensitive $\left(\mathrm{Ap}^{\mathrm{S}}\right)$, indicating that most $\mathrm{Mu}$ particles in the lysate containing MudphoA did not contain pUC18 18 . We concluded that the separation of these antibiotic markers was probably caused by the transposition of MudphoA at the permissive temperature.

We adapted the scheme of Castilho et al. (1984) to test the ability of MudphoA to generate active phoA fusion proteins. In this scheme a donor strain is constructed carrying one copy each of MudphoA and the helper prophage Mucts. The target plasmid is then introduced into the donor strain, transposition is derepressed by raising the temperature, and the lysate generated by this process is used to transduce a Mu-immune recipient. In 
some transductants, homologous recombination between Mu sequences results in recovery of $\mathrm{Mu}$ insertions in the target plasmid (Castilho et al., 1984).

To prevent the formation of target plasmid multimers, which would be recombinationally unstable following $\mathrm{Mu}$ insertion, it was necessary to construct this donor strain in a recombination-deficient background. Accordingly, M8820Mucts was transduced with a P1 lysate made from strain JC10240 (recA56 srl::Tn10 (Tc $\left.{ }^{\mathrm{F}}\right)$ ) (Csonka and Clark, 1979), selecting for tetracycline resistance. The resulting strain, EA4, was purified and tested for the recA mutation by u.v. light sensitivity (Maniatis et al., 1982). Strain EA4 was then transduced by a M8820Mucts (pUCA::MudphoA) lysate, followed by selection for $\mathrm{Km}^{\mathrm{R}}$ lysogens in which MudphoA had inserted despite $\mathrm{Mu}$ immunity. While the frequency of obtaining these double Mu lysogens was low, one was selected, i.e. strain EA17. Chromosomal DNA from strains EA17 and EA4 and pUC184::MudphoA plasmid DNA was cut with HindIII and probed with radiolabelled pUC184::MudphoA. Comparison of the hybridizing fragments among these DNAs showed that EA17 contained the same Mu fragments as EA4 as well as fragments identical to pUC18 $:$ :MudphoA that were internal to MudphoA, but no fragments specific to the vector pUC18A. In addition, alkaline lysis preparations of EA17 produced no detectable plasmid DNA. We concluded from this evidence (data not shown) that EA17 contained chromosomal copies of the two Mu genomes.

\section{Isolation and expression of MudphoA fusions in $\beta$ - lactamase and the L. pneumophila mip gene}

The $\beta$-lactamase gene on pBR322 encodes a periplasmic protein (Bolivar et al., 1977). TnphoA insertions into this gene generate active phoA fusions (Manoil and Beckwith, 1985). To test for MudphoA fusions to this gene, pBR322 was introduced into EA17 by transformation. One transformant was induced for phage growth and transposition at $42^{\circ} \mathrm{C}$. The lysate from this strain was used to transduce

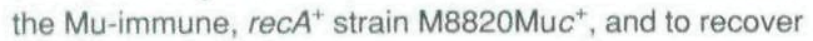
potential MudphoA insertions. The Mu-immune strain M8820Muc $\mathrm{C}^{+}$was used as a recipient to reduce the likelihood of additional transpositions by the incoming MudphoA.

Table 2 shows the frequencies of the various phenotypes generated by pBR322::MudphoA insertion. The frequency of insertions into the $A p^{A}$ and $T c^{R}$ genes was lower than expected given the size of these regions on pBR322 (Sutcliffe, 1979). About 14\% of the MudphoA insertions in the $\mathrm{Ap}^{R}$. gene were $\mathrm{PhoA}^{+}$, which is close to the expected frequency $(16.7 \%)$ of MudphoA in-frame translational fusions (two orientations, three reading frames)

We next isolated fusions to an L. pneumophila gene,
Table 2. Phenotype of $\mathrm{Km}^{\text {R }}$ transductants with a Mu lysate from EA17(pBR322).

\begin{tabular}{lcr}
\hline Phenotype & Number counted & $\%$ \\
\hline$A p^{R}, T c^{R}, P^{-} A^{-}$ & 995 & 94.5 \\
$A p^{R}, T c^{S}, P h o A^{-}$ & 20 & 2.0 \\
$A p^{S}, T c^{R}, P h o A^{-}$ & 30 & 3.0 \\
$A p^{S}, T c^{R}, P h o A^{-}$ & 5 & 0.5 \\
\hline
\end{tabular}

mip, whose product is an outer membrane protein. When mip is cloned on an $E$. coli replicon, its protein product localizes to the E. coli surface (Engleberg et al., 1984a,b). A plasmid carrying a cloned copy of mip, pNC31.5 (Cianciotto et al., 1989), was introduced into EA17 by transformation. The resulting strain was induced for phage growth and two mip::MudphoA insertions having alkaline phosphatase activity were isolated following transduction of M8820Muc ${ }^{+}$. We introduced the mip::MudphoA fusion plasmids into L. pneumophila strain AA100 by electroporation. Transformants expressed PhoA activity on XPcontaining agarose plates (see the Experimental procedures), confirming that MudphoA fusions can be expressed in L. pneumophila as well as in E. coli.

To confirm that the alkaline phosphatase activity of these strains resulted from fusions to MudphoA and not from other events (such as constitutive phosphatase mutations), strain $\mathrm{CC} 118$, an E. colistrain that has a phoA deletion (phoA $\triangle 20$ ) (Manoil and Beckwith, 1985) was transformed with each of the presumed $\mathrm{PhoA}^{+}$plasmids. All of the resulting $\mathrm{CC} 118$ strains acquired alkaline phosphatase activity, demonstrating that the $\mathrm{PhoA}^{+}$phenotype was of plasmid origin.

The insertion points of MudphoA in both the $\beta$-lactamase gene and the mip gene were mapped and the sizes of the expected fusion proteins were estimated. Several bla::MudphoA insertions mapped in a region approximately $300 \mathrm{bp}$ from the beginning of the bla gene while other bla::MudphoA insertions mapped approximately $400,500,600$ and 800 bp into the bla gene. Of the two mip::MudphoA insertions, one mapped approximately $200 \mathrm{bp}$ upstream and the other $100 \mathrm{bp}$ downstream from a unique HindIII site located near the $C$-terminal end of mip (Engleberg et al., 1989).

We also analysed whole cell lysates of the $\mathrm{CC} 118$ strains containing plasmids with MudphoA fusions by immunoblotting with anti-alkaline phosphatase antibody and anti-Mip monoclonal antibody (12F4) (Cianciotto et al., 1989). Anti-alkaline phosphatase antibody produced reactive bands in each of the bla::MudphoA samples and the molecular weights of these protein species closely approximated the predicted sizes based on restriction mapping. In the case of the mip::MudphoA samples, both anti-alkaline phosphatase and anti-mip antibody reacted 
A

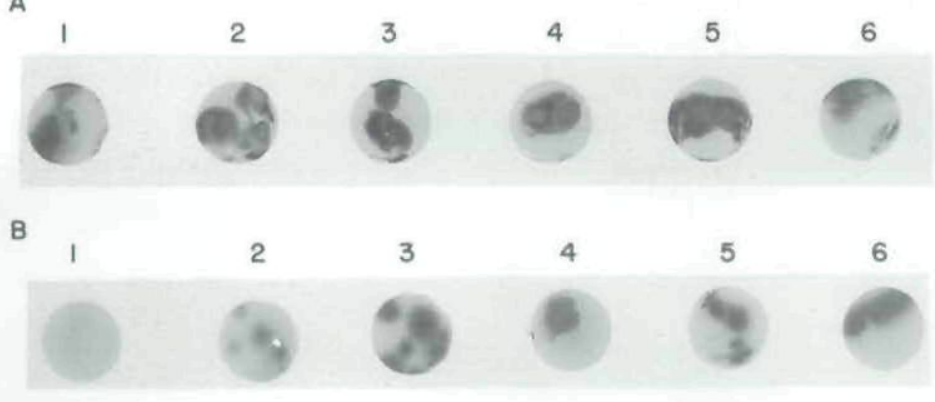

Fig. 3. Detection of alkaline phosphatase activity on XP-agarose plates. In panel A, the XP-agarose has been adjusted to a pH of 7.0 and in panel $B$ to a pH of 11.0. In both panels, disc 1 is AA103, discs 2-5 are PhoA ${ }^{*}$, MudphoA insertion mutants $A A 110, A A 111$ AA112, and $A A 113$ and disc 6 is AA114, a PhoA* L. pneumophila carrying pNC31.5::MudphoA (mip:MudphoA). At pH 7, all of the strains, including $A A 103$ which lacks a MudphoA insertion, produce a blue colour due to the native $L$. pneumophila phosphatase (Muller, 1981). At pH 11.0, only the $L$. pneumophila strains with MudphoA insertions that resulted in $E$. coli alkaline phosphatase activity produce a blue colour. with the same bands, which also closely approximated the predicted molecular weights (data not shown). We concluded from these observations that MudphoA insertions into the bla and mip genes resulted in the formation of stable fusion proteins.

\section{Construction of a delivery vector, pTLP7::MudphoA}

For delivery of MudphoA into Legionella, we constructed a vector, PTLP7, that has both selectable and counterselectable genetic markers. We included the broad-hostrange plasmid pRK2 origin of transfer sequence, oriT (Guiney and Yakobson, 1983), to mobilize the vector into $L$. pneumophila using transfer functions provided in trans by a helper conjugational plasmid, pRK212.1 (Figurski et al., 1976). Counterselection of the delivery vector facilitates the isolation of chromosomal MudphoA insertions since any kanamycin-resistant transconjugants remaining after counterselection must be due to non-vector, or chromosomal, copies of MudphoA. We chose the $s a c B$ allele of Bacillus subtilis as the counterselectable marker (Gay et al., 1985). The sacB gene encodes a sucrose-inducible levansucrase that synthesizes levans, branched polymers of fructose. Synthesis of these polymers within the periplasm of $L$. pneumophila is lethal. Thus, $L$, pneumophila carrying this gene will not grow on buffered charcoal yeast extract (BCYE) supplemented with sucrose, and only cells that have lost the allele are able to survive (Cianciotto et al., 1988).

Strain EA17 was transformed with pTLP7 and MudphoA insertions into the plasmid were isolated. One of these pTLP7::MudphoA plasmids was purified, and the insertion was mapped to ensure that MudphoA had not inserted into a strategically important sequence, such as the sacB gene. pRK212.1 and pTLP7::MudphoA were then introduced by transformation into strain $\chi 2981$, a diaminopimelic acid (dap) auxotroph, since exclusion of diaminopimelic acid from selective BCYE medium counterselects the $E$. coli donor strains in conjugal matings.
Isolation of MudphoA insertions with alkaline

phosphatase activity in L. pneumophila

We used tri-parental conjugative matings to introduce pTLP7::MudphoA into L. pneumophila (Engleberg et al., 1988), and screened the resulting $\mathrm{Km}^{R}$ exconjugants for phoA activity. Strain AA103 was used as the recipient strain because it generates higher frequencies of transconjugants in such matings (Cianciotto et al., 1988). We did not subject the mating mixtures to sucrose counterselection, since simultaneous selection for $\mathrm{Km}^{\mathrm{R}}$ and sucrose counterselection reduced the total number of transconjugants by about 10 -fold. Plasmid counterselection could be more efficiently applied after isolation of $\mathrm{PhoA}^{+}$transductants. In a typical experiment, we screened between $1000-2000 \mathrm{Km}^{\mathrm{F}}$ transductants. The frequency of $\mathrm{PhoA}^{+}$isolates was about $0.1 \%$. Some positive colonies developed colour more rapidly than others, probably indicating different levels of phosphatase expression.

Figure 3 demonstrates the PhoA screening assay using XP-agarose indicator plates. At pH 7, both $\mathrm{PhoA}^{+}$and $\mathrm{PhoA}^{-}$strains exhibited phosphatase activity, seen as an intense blue colour on the XP-agarose indicator medium (Fig. $3 \mathrm{~A}$ ). By raising the $\mathrm{pH}$ of the medium, the background activity produced by the native $L$. pneumophila phosphatase was minimized (Fig. 3B, disc 1), whereas strains with $\mathrm{PhoA}^{+}$gene fusions continued to produce a blue colour (Fig. 3B, discs 2-7). Strain AA114 (mip::MudphoA) was included to show the expression of a MudphoA gene fusion to a known L. pneumophila surface protein. $\mathrm{PhoA}^{+}$strains $\mathrm{AA} 110$ and $\mathrm{AA} 112$ consistently produce a blue colour that is less intense than the colour produced by AA111, AA113 or AA114.

The $\mathrm{PhoA}^{+}$L. pneumophila isolates were also analysed by Southern hybridization (Fig. 4). Figure 4, panel A shows genomic DNA from four $\mathrm{PhoA}^{+}$strains, digested with HindIII and probed with pTLP7:: MudphoA. The MudphoA insertions are different in each strain, since the 


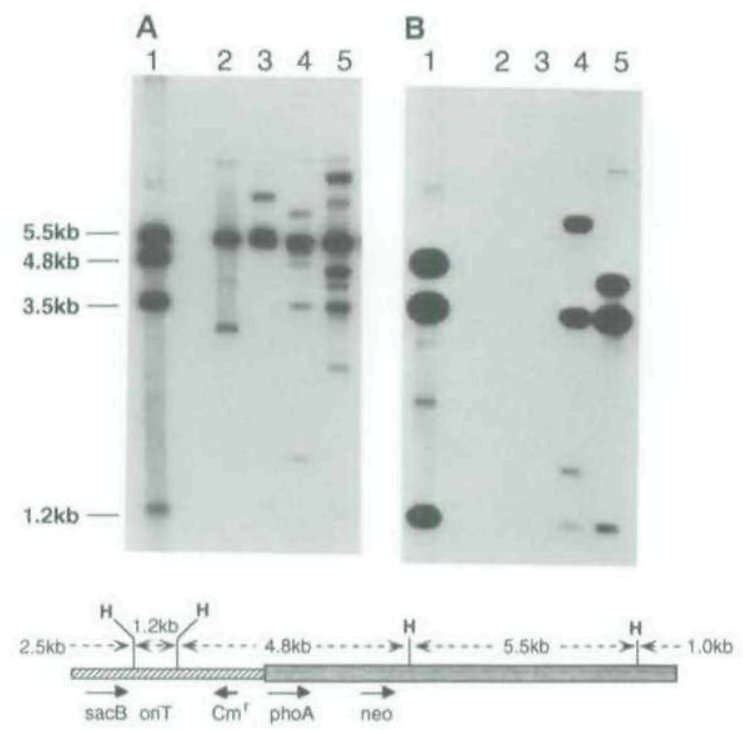

Probe $\mathrm{A}$

Probe B

Fig. 4. Southern hybridization of L. pneumophila having MudphoA insertions. Lane 1 (control), PTLP7:MudphoA; lane 2, AA110; lane 3, AA111: lane 4, AA112; lane 5, AA113. The HindIII restriction map of PTLP7::MudphOA is shown below the photographs. The thinner, diagonally striped segment corresponds to PTLP7 vector sequences and the thicker, darkly shaded segment is MudphoA, inclusive of the Mu right and left ends. The corresponding sequences used as probes are indicated below the map. In panel A, the fitter was probed with PTLP7::MudphoA (probe A). In panel B, the filter was washed tree of probe A and reprobed with pTLP7 (probe B) to identify vector-specific fragments. Note that all of the samples in panel A have a 5.5 kilobase $(\mathrm{kb})$ internal MudphoA fragment. Note that AA110 and AA111 have only one MudphoA insertion each, and they are free of vector sequences. AA112 and AA113 have retained vector sequences as well as transpositions. The high molecularweight band in the AA110, AA111, AA112, and AA113 samples (lanes $2-5$ ) is presumably pRK212.1 which has a unique Hindill site and shares the oriT sequence with the probe (Figurski et al., 1976).

sizes of the junctional bands, determined by the positions of flanking genomic HindIII sites, are different. Each MudphoA insertion would therefore produce three bands, one corresponding to the $5.5-\mathrm{kb}$ internal fragment, and two variable junction fragments. AA111 (Fig. 4, lane 3) has three fragments, indicating a single insertion. AA110 (Fig. 4, lane 2) has two fragments that react strongly; one corresponds to the 5.5-kb internal fragment. The third fragment is probably the weaker band at approximately $4.0 \mathrm{~kb}$. AA112 and AA113 (Fig. 4, lanes 4 and5) have multiple insertions. By comparing lane 1 ( $p$ TLP7::MudphoA) with $\mathrm{AA} 112$ and $\mathrm{AA} 113$ (Fig. 4, lanes 4 and 5) it appears that these last two strains contain vector-specific fragments. Since we did not subject the matings to counterselection to eliminate the vector, the presence of these bands is not surprising. The spontaneous loss of the vector in AA110 and AA111 is consistent with the behaviour of ColE1 replicons in L. pneumophila (Engleberg et al., 1988). To confirm this interpretation, the same filter was probed with pTLP7 (Fig. 4B). Vector sequences were present in strains AA112 and AA113 (lanes 4,5) but not in $A A 110$ and AA111. Moreover, the vector fragments in AA112 and AA113 were of unexpected sizes, indicating some rearrangement of the vector sequences.

\section{Detection of MudphoA fusion proteins}

To detect the presence of fusion proteins in the PhoA $\mathrm{A}^{+}$. pneumophila strains, we performed immunoblotting of whole cell lysates with anti-alkaline phosphatase antibody (kind gift of Dr David A. Lowe)(Fig. 5). In each case, the antibody detected fusion proteins with apparent molecular weights greater than that of native alkaline phosphatase (Fig. 5, lane 1). The fusion proteins in strains AA111, AA113 and AA114 (lanes $4,6,7$ ) reacted more intensely than those in strains AA110 and AA112 (Fig. 5, lanes 3 and 5). This pattern of reactivity correlated with the differences in intensity of blue colour detected on XP. agarose indicator plates for these strains (Fig. 3). The smaller bands seen with most of the samples presumably correspond to alkaline phosphatase degradation products. These products also appear to be of different sizes and intensities, except for one fragment common to all the samples that is slightly smaller than the alkaline phosphatase monomer (Fig. 5, lane 1). Presumably this smaller, common band represents the product of the truncated phoA fragment used in MudphoA.

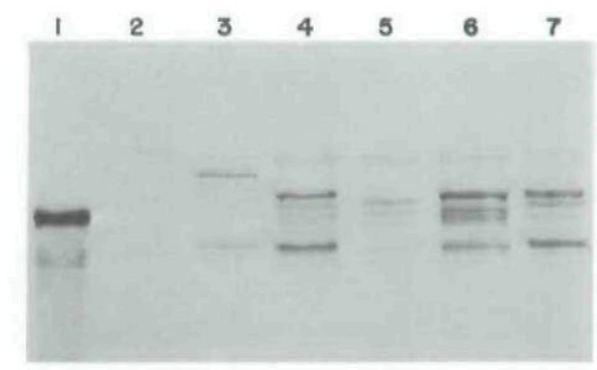

Fig. 5. Immunoblot of MudphoA fusion proteins with anti-PhoA serum. Whole cell lysates were prepared from $L$. pneumophila strains as described by Peariman et al. (1985), electrophoresed in a SDS-poly. acrylamide gel $(8 \%)$, transferred to nitrocellulose and reacted with antialkaline phosphatase antibody. Lane 1, denatured alkaline phosphatase; lane 2, AA103; lane 3, AA110; lane 4, AA111; lane 5, AA112; lane 6, AA113; lane 7, AA114. Note the absence of reactive protein in AA103 and the presence of strongly reacting bands in the other lanes which contain samples from $\mathrm{PhoA}^{+}$L. pneumophila strains. In lane 7, the mip::phoA fusion protein had a predicted size of $56 \mathrm{kDa}$ based on restriction enzyme analysis of the insertion site on pNC31.5. The other strains show variably sized bands at least one of which is larger than alkaline phosphatase monomer in each case. 

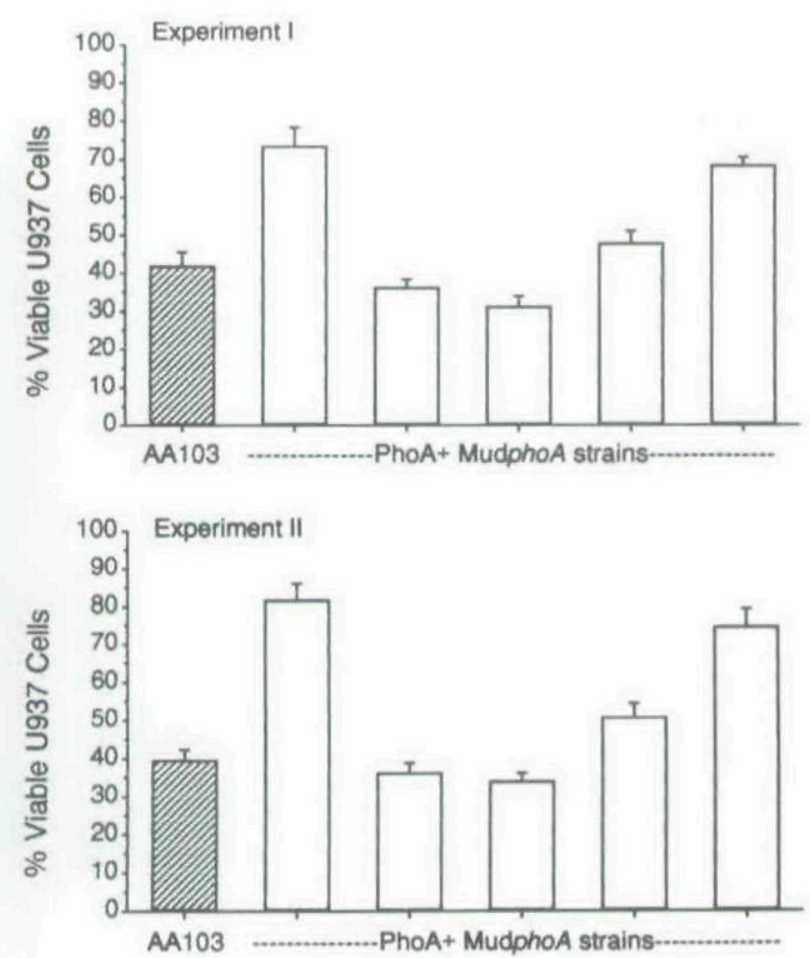

Fig. 6. Reproducible cytopathic effect of PhoA MudphoA mutants of $L$. pneumophila in a U937 cell assay. The two experiments shown were performed on separate days with separate cultures of bacteria and monocytic cells with inocula of $10^{6}$ bacteria. After $72 \mathrm{~h}$ at $37^{\circ} \mathrm{C}$, the U937 cell viability was determined by staining with $\mathrm{MTT}(\%$ viable U937 cells = mean $\mathrm{OD}_{550}$ for each strain $\div$ mean $\mathrm{OD}_{550}$ of uninoculated, control U937 cell wells $\times 100$ ). AA103 is the virulent, parent strain and contains no MudphoA insertions. PhoA $A^{+}$mutants studied were (from left to right): AA110, AA111, AA112, AA113, and AA118.

Relative infectivities of PhoA ${ }^{+}$MudphoA L. pneumophila mutants in U937 cell assays

MudphoA contains the temperature-sensitive repressor allele $c t s(62)$ that regulates transposition in a temperature-dependent manner. The utility of MudphoA in identifying virulence factors in L. pneumophila (or any other pathogen) depends upon the effect, if any, on bacterial cell viability of growth at $37^{\circ} \mathrm{C}$. That is, if Mu transposition is sufficiently derepressed at $37^{\circ} \mathrm{C}$ to kill MudphoA-containing bacteria, all strains having functional transposons would appear to be attenuated in virulence assays conducted at this temperature. Since we intend to screen PhoA $A^{+}$MudphoA L. pneumophila mutants for an in vitro U937 cell cytopathicity effect (CPE) at $37^{\circ} \mathrm{C}$ (Pearlman et al., 1988), it is important to insure that any observed reduction in bacterial virulence is caused by insertional inactivation of a virulence-related gene rather than by bacterial death resulting from derepressed Mu transposition.

To address this concern, we tested the plating efficiencies of $\mathrm{PhoA}^{+}$MudphoA L. pneumophila mutant strains at $30^{\circ} \mathrm{C}$ and $37^{\circ} \mathrm{C}$. In all strains examined, there was no significant difference in the cfu numbers of individual MudphoA L. pneumophila mutant strains grown at either temperature (data not shown). Next, we conducted several independent experiments to compare $\mathrm{PhoA}^{+}$MudphoA $L$. pneumophila mutants and the parent strain, AA103, in the cytopathicity assay using U937 cells. Figure 6 shows the results of two of these assays. Clearly, this screening assay detects reproducible differences between the $\mathrm{PhoA}^{+}$MudphoA mutants; some are as virulent as the parent strain, whereas two mutants were significantly attenuated. These experiments suggest that any secondary transposition induced by growth at $37^{\circ} \mathrm{C}$ is not sufficient to affect the infectivity of these strains, as measured by the U937 cell assay.

\section{Discussion}

We described construction of MudphoA, a derivative of Mu dll4041 (Castilho et al., 1984) that can be used to generate alkaline phosphatase gene fusions in vivo. To generate a $\mathrm{PhoA}^{+}$fusion, MudphoA must insert into a gene with an amino-terminus encoding a signal peptide, since alkaline phosphatase is normally active only when it is exported from the cytoplasm (Michaelis et al., 1983; Hoffman and Wright, 1985; Manoil and Beckwith, 1985). Insertions of MudphoA in several different positions in the gene for the periplasmic $\beta$-lactamase protein gave fusion proteins with alkaline phosphatase activity. Based on restriction mapping, many of these insertions were in the same region of the $\beta$-lactamase gene, possibly indicating non-random Mu-insertion activity or lethality of certain insertions (Castilho et al., 1984; Hall and Silhavy, 1981). Two different insertions in the gene for the membranelocalized Mip protein of $L$. pneumophila similarly also resulted in enzyme activity, both in E. coli and in L. pneumophila.

It is reasonable to assume that detection of $\mathrm{PhoA}^{+}$ MudphoA fusions in L. pneumophila will identify genes encoding signal peptides, a hallmark of proteins that are secreted from the cytoplasm. Secreted proteins are those most likely to be involved in mediating $L$. pneumophila pathogenesis; therefore we can use MudphoA to identify and to mutate the genes encoding these proteins. An advantage of using MudphoA is that chromosomal insertions can be selected in vivo under different environmental conditions, thereby allowing identification of selectively expressed genes that may be important for virulence, but are otherwise difficult to identify using in vitro methods. For example, one can select for $\mathrm{PhoA}^{+}$MudphoA insertions at lower temperatures, under iron limitation, oxygen stress or low/high osmolarity - conditions known to affect expression of virulence determinants in other pathogenic bacteria. 
Mintz and Shuman reported the ability of Mu to transpose to many sites within the Legionella genome, by generating insertion mutations and lac gene fusions (Mintz and Shuman, 1987). We have obtained similar results with MudphoA. All of the fusion strains isolated to date stably maintain the $\mathrm{PhoA}^{+}$phenotype after several passages on $\mathrm{BCYE} / \mathrm{Km}$ media.

Fusions generated by MudphoA insertion contain 38 extra amino acids resulting from translation of the $\mathrm{Mu}$ right end sequence (Fig. 2). The presence of this extra sequence had no effect on lac fusion protein expression (Castilho et al., 1984); however, its effect on the secretion and dimerization of $\mathrm{PhoA}^{+}$fusion proteins was unpredictable. Our results indicate that this linking peptide does not prevent expression of the enzyme, although proteolytic cleavage in this region seems to occur frequently, judging by the products seen in Fig. 5 .

To screen $L$. pneumophila strains for the presence of $E$. coli alkaline phosphatase activity, we developed an in situ assay for PhoA activity. This assay helped us overcome several technical difficulties associated with screening for PhoA activity in L. pneumophila. For example, the black colour of BCYE medium interferes with blue colour detection of PhoA activity, and incorporation of the colour indicator substrate, $X P$, into $B C Y E$ is toxic to $L$. pneumophila. In addition, the native phosphatase activity of $L$. pneumophila masks the $E$. coli phosphatase activity at $\mathrm{pH}$ levels that permit bacterial growth; however, this background activity becomes insignificant at a $\mathrm{pH}$ of about 11.0.

The broad-host-range plasmid pRK212.1; which was used in this study to provide transfer functions, is itself transferred at a high frequency (Fig. 4). It is possible to eliminate this plasmid either by passage on non-selective media or by 'curing'. Its use may be avoided by introducing MudphoA from an E. coli strain carrying the Tra functions on the chromosome or by placing MudphoA on a conjugative 'suicide' vector (Engleberg et al., 1988; Miller, 1972; Keen et al., 1985). In any case, the presence of pRK212.1 in L. pneumophila confers no detectable change on intracellular infectivity (J. E. Rogers, unpublished observations). In some cases (Fig. 4B) the delivery vector, or derivatives of it, is present in the fusion strains. The presence of the apparently modified vector fragments suggests possible Mu-induced inversions or deletions (Pato, 1989). Sucrose counterselection effectively eliminates sacB-containing plasmids from $L$. pneumophila, but it is not known how this counterselection will effect these unexplained derivatives (Cianciotto et al., 1988).

We have isolated several $\mathrm{PhoA}^{+}$L. pneumophila transconjugants, and we have found two of these strains (AA110, AA118) to be significantly attenuated in a U937 cell-infectivity assay. However, certain biological features of the transposon impose limitations on the use of Mud-
phoA for identification of virulence genes. For example, since derepression of $\mathrm{Mu} \mathrm{cts}(62)$ phage transposition can be reliably induced at $42^{\circ} \mathrm{C}$, it is unlikely that animal challenge experiments could be performed using MudphoA mutants. The febrile temperatures would probably induce enough secondary transposition to limit the growth and survival of $L$. pneumophila strains that carry MudphoA. A more immediate concern is that some leakiness of the $\mathrm{Mu}$ cts(62) might occur at the lower, semi-permissive temperature of $37^{\circ} \mathrm{C}$ and result in a similar bacterial attrition in the U937 cell-infectivity assay. At the very least, passage of the bacteria at $37^{\circ} \mathrm{C}$ might be expected to result in a heterogeneous population of bacteria containing multiple copies of the transposon in diverse chromosomal locations.

To limit secondary transpositions, we routinely maintain and cultivate all L. pneumophila (MudphoA) strains at $30^{\circ} \mathrm{C}$ or store them at $-70^{\circ} \mathrm{C}$. All bacterial inocula for the U937 cell assay are grown at $30^{\circ} \mathrm{C}$, and any bacteria isolated from cell cultures (at $37^{\circ} \mathrm{C}$ ) are discarded. By exercising this precaution, we have shown that most $\mathrm{PhoA}^{+} L$. pneumophila strains are as cytopathic as the parental strain in the U937 cell assay. This normal level of cytopathicity has been demonstrated in repeated experiments on different days, using different cultures of the $L$. pneumophila test strains and different batches of differentiated U937 cells. In the same experiments, the two PhoA ${ }^{+}$ mutants that were attenuated in our initial screening assay were comparably attenuated in all subsequent assays. We conclude from this experience that $L$. pneumophila does not become attenuated in the U937 cell assay simply because of the presence of either MudphoA or a $\mathrm{PhoA}^{+}$gene fusion. Furthermore, the attenuated $\mathrm{PhoA}^{+}$mutants are likely to acquire this phenotype as a result of transposon-induced mutations in virulencerelated genes.

The multiplicity of MudphoA transpositions in L. pneumophila presents a second potential problem in characterizing the phenotype of attenuated mutants, since most transconjugants contain more than one copy of the transposon (typically $1-4$ copies). Although it is likely that only one of these copies is responsible for the $\mathrm{PhoA}^{+}$activity, it is by no means certain that the fused gene is also responsible for the attenuated phenotype. For this reason (and because of the additional concern about possible secondary transpositions, outlined above), all mutations of interest will eventually have to be isolated by molecular cloning and then reintroduced into a wild-type strain by homologous recombination. This manoeuvre will determine whether the $\mathrm{PhoA}^{+}$gene fusion or some other MudphoA insertion is responsible for the attenuated phenotype. In addition, a reconstructed strain will not have a functional transposon and could be used for animal challenge studies and extended phenotypic characterization. 


\section{Experimental procedures}

\section{Bacterial strains, plasmids and growth conditions}

The E. coli and L. pneumophila bacterial strains used in this study are listed in Table 1. Strain BAC101, obtained from M. J. Casadaban, was our source for Mu dll4041 (Casadaban and Chou, 1984). Strain CC118 is an E. coli phoA deletion strain obtained from C. Manoil (Manoil and Beckwith, 1985). L. pneumophila strain AA100 is a serogroup 1 clinical isolate, $103 \mathrm{~b}$ (Los Angeles), and AA103 is a derivative of AA100 that is a high-frequency conjugal recipient (Engleberg et al., 1988; Cianciotto et al., 1988).

Plasmids used include pUC18 18 , which was derived in this lab. from pUC18 by deletion of the small Pvull fragment containing multiple cloning sequences (Yanisch-Perron et al., 1985). pRK212.1 is a deletion derivative of pRK2 that is $\mathrm{Km}^{\mathrm{s}}$ (Figurski et al., 1976).

E. coli strains were routinely cultured from frozen stocks onto Luria-Bertani (LB) medium or in LB broth containing antibiotics as required. Antibiotics were used at the following concentrations; kanamycin $(\mathrm{Km}) 25 \mathrm{gg} \mathrm{ml}^{-1}$, tetracycline $(\mathrm{Tc})$ $15 \mu \mathrm{g} \mathrm{ml}^{-1}$, chloramphenicol $(\mathrm{Cm}) 20 \mathrm{~g} \mathrm{ml}^{-1}$ and ampicillin (Ap) $50 \mu \mathrm{g} \mathrm{ml}^{-1}$. L. pneumophila strains were also cultured from frozen stocks onto BCYE medium, containing $25 \mu \mathrm{g} \mathrm{ml}^{-1}$ kanamycin as required.

\section{Genetic and DNA manipulations}

Chromosomal DNA was prepared from E. coli and L. pneumophila as described by Ausubel et al. (1987). Plasmid DNA preparation, DNA cloning and Southern hybridizations were performed using standard methods (Maniatis et al., 1982). DNA restriction enzymes were used as suggested by the manufacturers. ${ }^{32} \mathrm{P}$-labelled DNA probes were made with the multi-prime kit (Amersham).

Cloning and isolation of recombinant plasmids were done in E. coli strain HB101. Transformation of temperature-sensitive MudphoA-containing plasmids was performed by electroporation, using a Bio-Rad Gene Pulser apparatus (Bio-Rad) or by the method of Chung et al. (1989). Other transformations were by standard procedures (Maniatis et al., 1982). E. coli and $L$. pneumophila cells were prepared for electroporation by the method of Dower et al. (1988). Methods for handling bacteriophage $\mathrm{Mu}$ and $\mathrm{P} 1$-mediated transduction of $E$. coli were as described by Bukhari and Ljungquist (1977) and Silhavy et al. (1984).

Tri-parental bacterial conjugations for introduction of MudphoA into L. pneumophila were performed as described by Engleberg et al. (1988), with minor modifications. Mating mixtures of AA103, $\chi 2981$ (pRK212.1) and $\chi 2981$ (pTLP7::Mud$p h o A)$ were incubated on nitrocellulose filters on the surface of $\mathrm{BCYE}$ medium for $6 \mathrm{~h}$ at $30^{\circ} \mathrm{C}$. The mating mixtures were then resuspended and plated onto $B C Y E / K m$ at appropriate dilutions to obtain 100-200 colonies per plate. The plates were incubated for an average of 6 days at $30^{\circ} \mathrm{C}$, until the colonies had reached optimum size for transfer to XP-agarose plates.

\section{Plate assays for detection of PhoA fusions}

For detection of alkaline phosphatase activity in $E$. coli strains resulting from MudphoA insertions, we incorporated 5-bromo- 4-chloro-3-indolyl phosphate (XP) at a concentration of $40 \mu \mathrm{g}$ $\mathrm{ml}^{-1}$ into the selective LB media. The high phosphate concentration in LB medium effectively suppressed expression of the native $E$. coli alkaline phosphatase, allowing easy detection of PhoA activity resulting from MudphoA fusion proteins.

This strategy fails to detect $E$. coli alkaline phosphatase activity in L. pneumophila because the black background colour of BCYE prevents visualization, XP inhibits $L$. pneumophila growth, and L. pneumophila produces a broad $\mathrm{pH}$ range $(\mathrm{pH} 4-8)$ phosphatase activity that is not inhibited by high phosphate concentration (Muller, 1981). To circumvent these technical problems, we developed XP-containing agarose plates for detection of $\mathrm{PhoA}^{+}$L. pneumophila strains. The XP-agarose plates were made as follows: $1.5 \mathrm{~g}$ of electrophoresis grade agarose was mixed with $100 \mathrm{ml}$ of a 0.1 molar solution of 3-(cyclohexylamino)-1-propanesulphonic acid (CAPS) buffer (Sigma). The suspension was then brought to $\mathrm{pH} 11.0$ with $10 \mathrm{M} \mathrm{NaOH}$ and heated to dissolve the agarose completely. After the solution cooled to approximately $40^{\circ} \mathrm{C}$, XP (40 mg ml-1 in dimethylformamide) was added at a concentration of $40 \mathrm{\mu g} \mathrm{ml}^{-1}$ and $10 \mathrm{ml}$ of the XP-agarose solution was allowed to solidify in Petri plates.

L. pneumophila $\mathrm{Km}^{R}$ transconjugant colonies were lifted onto nitrocellulose filters, and the filters were applied colonyside-down onto the surface of XP-agarose plates. PhoA colonies were generally apparent after $1-2 \mathrm{~h}$ incubation at room temperature. In each experiment $\mathrm{PhoA}^{+}$and $\mathrm{PhoA}^{-}$control strains were included on the filters to assess the development of blue colour because of $E$. coli alkaline phosphatase activity. When $\mathrm{PhoA}^{+}$colonies were detected, master plates were re-incubated at $30^{\circ} \mathrm{C}$ until good colony growth was achieved (about 3 days). The $\mathrm{PhoA}^{+}$colonies were identified, picked, and streaked for purity on BCYE/Km media. After reisolation, strains were always re-tested on fresh XP-agarose plates. The XP-agarose plates were efficient at detecting alkaline phosphatase activity as long as the plates were used when freshly made. After about $48 \mathrm{~h}$ the plates began to lose specificity, probably owing to a spontaneous reduction in $\mathrm{pH}$.

\section{Antibodies and immunoassays}

To detect alkaline phosphatase fusion proteins, whole cell lysates of strains were prepared and reacted with anti-alkaline phosphatase antibody. L. pneumophila strains were grown on plates for $48-72 \mathrm{~h}$ at the appropriate temperature. Cells were suspended in water to an optical density at $550 \mathrm{~nm}\left(\mathrm{OD}_{550}\right)$ of 1.0 and samples were boiled for $5 \mathrm{~min}$ in the presence of SDS and 2-mercaptoethanol (Pearlman et al., 1985). The lysates were centrifuged briefly to pellet debris, and equal amounts were electrophoresed in an $8 \%$ discontinuous SDS-polyacrylamide gel. Alkaline phosphatase (Sigma) was treated as above and included in the immunoassays. The separated proteins were transferred to nitrocellulose, and immunoblots were prepared as previously described (Cianciotto et al., 1989). Horseradish peroxidase-conjugated goat anti-rabbit antibody (Cappel Industries) was used as the secondary antibody and diaminobenzidine (Sigma) was used as the colour indicator substrate.

\section{U937 cell cytopathicity}

Determination of the cytopathic effects of $L$. pneumophila 
strains was performed as described by Pearlman et al. (1988) with slight modifications. Bacteria grown on either BCYE or $\mathrm{BCYE} / \mathrm{Km}$ at $30^{\circ} \mathrm{C}$ were added to adherent U937 cells in 96well plates ( $10^{5}$ cells per well), and the infection was allowed to proceed at $37^{\circ} \mathrm{C}$ for $72 \mathrm{~h}$. The infected monolayers were neither washed nor treated with antibiotics during this incubation. At the end of the incubation, the U937 cell-cytopathic effect was determined as previously described. The vital stain tetrazolium salt 3-(4,5-dimethyl thiazol-2-yl)-2,5-diphenyl tetrazolium bromide (MTT; Sigma) was used to distinguish live from dead U937 cells. In all experiments, a set of eight replicate infections for each bacterial strain was examined. The lowest and highest $O_{550}$ readings were eliminated from the set of values used to calculate an average and standard deviation.

\section{Acknowledgements}

We thank Dr David A. Lowe for his kind gift of anti-alkaline phosphatase antibody, and M. J. Casadaban, C. Manoil, B. Bachmann, R. Curtiss III, and J. E. Rogers for strains. This work was supported by Public Health Service grants RO1 Al26232 (to N.C.E.), RO1 Al24731 (to B.I.E.). M. A. Albano is supported by NIH training grant 5 T32 Al07360.

\section{References}

Ausubel, F.M., Brent, R., Kingston, R.E., Moore, D.D., Seidman, J.G., and Struhl, K. (1987) Current Protocols in Molecular Biology. New York: John Wiley and Sons.

Beck, E., Ludwig, G., Auerswald, E.A., Reiss, B., and Schaller, $H$. (1982) Nucleotide sequence and exact localization of the neomycin phosphotransferase gene from transposon Tn5. Gene 19: 327-336.

Bolivar, F., Rodriguez, R.L., Greene, P.J., Betlach, M.C., Heyneker, H.L., and Boyer, H.W. (1977) Construction and characterization of new cloning vehicles. II. A multipurpose cloning system. Gene 2: 95-113.

Broome-Smith, J.K., Tadayyon, M., and Zhang, Y. (1990) Betalactamase as a probe of membrane protein assembly and protein export. Mol Microbiol 4: 1637-1644.

Bukhari, A.I., and Ljungquist, E. (1977) Bacteriophage Mu: methods for cultivation and use. In DNA Insertion Elements, Plasmids and Episomes. Bukhari, A.I., Shapiro, J.A., and Adhya, S.L. (eds). Cold Spring Harbor, New York: Cold Spring Harbor Laboratory Press, pp. 749-756.

Casadaban, M.J., and Chou, J. (1984) In vivo formation of gene fusions encoding hybrid beta-galactosidase proteins in one step with a transposable $\mathrm{Mu}$-lac transducing phage. Proc Natl Acad Sci USA 81: 535-539.

Castilho, B.A., Olfson, P., and Casadaban, M.J. (1984) Plasmid insertion mutagenesis and lac gene fusion with mini-mu bacteriophage transposons. J Bacteriol 158: 488-495.

Chang, C.N., Kuang, W.J., and Chen, E.Y. (1986) Nucleotide sequence of the alkaline phosphatase gene of Escherichia coli. Gene 44: 121-125.

Chung, C.T., Niemela, S.L., and Miller, R.H. (1989) One-step preparation of competent Escherichia coli: transformation and storage of bacterial cells in the same solution. Proc Natl Acad Sci USA 86: 2172-2175.

Cianciotto, N.P., Long, R., Eisenstein, B.I., and Engleberg, N.C. (1988) Site-specific mutagenesis in Legionella pneumophila by allelic exchange using counterselectable ColE1 vectors. FEMS Microbiol Lett 56: 203-208.
Cianciotto, N.P., Eisenstein, B.I., Mody, C.H., Toews, G.B., and Engleberg, N.C. (1989) A Legionella pneumophila gene encoding a species-specific surface protein potentiates the initiation of intracellular infection. Infect Immun 57: 12551262.

Csonka, L.N., and Clark, A.J. (1979) Deletions generated by the transposon $\mathrm{Tn} 10 \mathrm{in}$ the $\mathrm{srl}$ recA region of the Escherichia coli K-12 chromosome. Genetics 93: 321-343.

Dower, W.J., Miller, J.F., and Ragsdale, C.W. (1988) High efficiency transformation of $E$. coli by high voltage electroporation. Nucl Acids Res 16: 6127-6145.

Dreyfus, L.A., and Iglewski, B.H. (1985) Conjugation-mediated genetic exchange in Legionella pneumophila. J Bacteriol 161: $80-84$.

Dreyfus, L.A. and Iglewski, B.H. (1986) Purification and characterization of an extracellular protease of Legionella pneumophila. Infect Immun 51: 736-743.

Engleberg, N.C., Drutz, D.J., and Eisenstein, B.I. (1984a) Cloning and expression of Legionella pneumophila antigens in Escherichia coli. Infect Immun 44: 222-227.

Engleberg, N.C., Pearlman, E., and Eisenstein, B.I. (1984b) Legionella pneumophila surface antigens cloned and expressed in Escherichia coli are translocated to the host cell surface and interact with specific anti-Legionella antibodies. J Bacteriol 160: 199-203.

Engleberg, N.C., Cianciotto, N.P., Smith, J., and Eisenstein, B.I. (1988) Transfer and maintenance of small, mobilizable plasmids with ColE1 replication origins in Legionella pneumophila. Plasmid 20: 83-91.

Engleberg, N.C., Carter C., Weber D.R., Cianciotto N.P., Eisenstein B.I. (1989) DNA Sequence of mip, a Legionella pneumophila gene associated with macrophage infectivity. Infect Immun 57: 1263-1270

Engleberg, N.C., Howe, D.C., Rogers, J.E., Arroyo, J., and Eisenstein, B.I. (1991) Characterization of a Legionella pneumophila gene encoding a lipoprotein antigen. Mol Microbiol 5: 2021-2029

Figurski, D., Meyer, R., Miller, D.S., and Helinski, D.R. (1976) Generation in vitro of deletions in the broad host range plasmid RK2 using phage $\mathrm{Mu}$ insertions and a restriction endonuclease. Gene 1: 107-119.

Gay, P., Le Coq, D., Steinmetz, M., Berkelman, T., and Kado, C.I. (1985) Positive selection procedure for entrapment of insertion sequence elements in Gram-negative bacteria. $J$ Bacteriol 164: 918-921.

Guiney, D.G. and Yakobson, E. (1983) Location and nucleotide sequence of the transfer origin of the broad host range plasmid RK2. Proc Natl Acad Sci USA 80: 3595-3598.

Guyer, M.S., Reed, R.R., Steitz, J.A., and Low, K.B. (1981) Identification of a sex-factor-affinity site in $E$. coli as gamma delta. Cold Spring Harb Symp Quant Biol 45 (Pt 1): 135-140.

Hall, M.N., and Silhavy, T.J. (1981) Genetic analysis of the major outer membrane proteins of Escherichia coli. Annu Rev Genet 15: 91-142.

Hoffman, C.S., and Wright, A. (1985) Fusions of secreted proteins to alkaline phosphatase: an approach for studying protein secretion. Proc Natl Acad Sci USA 82: 5107-5111.

Kahmann, R., and Kamp, D. (1979) Nucleotide sequences of the attachment sites of bacteriophage Mu DNA. Nature 280: 247-250.

Keen, M.G., Street, E.D., and Hoffman, P.S. (1985) Broadhost-range plasmid pRK340 delivers Tn5 into the Legionella pneumophila chromosome. J Bacteriol 162: 1332-1335.

Maniatis, T., Fritsch, E.F., and Sambrook, J. (1982) Molecular 
Cloning. A Laboratory Manual. Cold Spring Harbor, New York: Cold Spring Harbor Laboratory Press.

Manoil, C., and Beckwith, J. (1985) TnphoA: a transposon probe for protein export signals. Proc Natl Acad Sci USA 82: 8129-8133.

Michaelis, S., Inouye, H., Oliver, D., and Beckwith, J. (1983) Mutations that alter the signal sequence of alkaline phosphatase in Escherichia coli. J Bacteriol 154: 366-374.

Miller, J.H. (1972) Experiments in Molecular Genetics. Cold Spring Harbor, New York: Cold Spring Harbor Laboratory Press.

Mintz, C.S. and Shuman, H.A. (1987) Transposition of bacteriophage $\mathrm{Mu}$ in the Legionnaires' disease bacterium. Proc Natl Acad Sci USA 84: 4645-4649.

Muller, H.E. (1981) Enzymatic profile of Legionella pneumophilia. J Clin Microbiol 13: 423-426.

Pato, M.L. (1989) Bacteriophage Mu. In Mobile DNA. Berg, D.E., and Howe, M.M. (eds). Washington, DC: American Society for Microbiology, pp. 23-51.

Pearlman, E., Engleberg, N.C., and Eisenstein, B.I. (1985)
Identification of protein antigens of Legionella pneumophila serogroup 1. Infect Immun 47: 74-79.

Pearlman, E., Jiwa, A.H., Engleberg, N.C., and Eisenstein, B.I. (1988) Growth of Legionella pneumophila in a human macrophage-like (U937) cell line. Microbial Pathog 5: 8795.

Silhavy, T.J., Berman, M.L., and Enquist, L.W. (1984) Experiments with Gene Fusions. Cold Spring Harbor, New York: Cold Spring Harbor Laboratory Press.

Sutcliffe, J.G. (1979) Complete nucleotide sequence of the Escherichia coli plasmid pBR322. Cold Spring Harb Symp Quant Biol 43 (Pt 1): 77-90.

Williams, A.E., Maskell, D.J., and Moxon, E.R. (1991) Relationship between intracellular survival in macrophages and virulence of Haemophilus influenzae type b. J Inf Dis 163: 13661369.

Yanisch-Perron, C., Vieira, J., and Messing, J. (1985) Improved $\mathrm{M} 13$ phage cloning vectors and host strains: nucleotide sequences of the M13mp18 and pUC19 vectors. Gene 33: 103-119. 
This document is a scanned copy of a printed document. No warranty is given about the accuracy of the copy. Users should refer to the original published version of the material. 